\title{
El desempeño fiscal municipal y su influencia en el crecimiento económico de los municipios colombianos
}

\author{
Municipal fiscal performance and its influence on the economic growth in Colombian municipalities
}

\author{
Alexander Carvajal ${ }^{1}$ y Oscar David Andrés Julián López-Camargo ${ }^{2}$ (i)
}

${ }^{1}$ Universidad Santo Tomás Tunja, Colombia. Email: alexander.carvajal@ usantoto.edu.co
${ }^{2}$ Universidad Santo Tomás Tunja, Colombia. Email:

Para citar este artículo: Carvajal, A. y López, C. O. (2020). El desempeño fiscal municipal y su influencia en el crecimiento económico de los municipios colombianos. Clío América, 14(28), 504-516. http://dx.doi.org/10.21676/23897848.4027

Recibido: 13 abril de 2020

Aceptado: 21 de septiembre de 2020

Publicado en línea: noviembre 20 de 2020

\section{Palabras clave:} modelos de econometría espacial; autocorrelació n espacial; desempeño fiscal municipal; actividad económica municipal; gobiernos municipales.

\section{Keywords:} models of spatial econometrics ; municipal economic activity; municipal fiscal performance; municipal governments ; spatial autocorrelati on.

\section{RESUMEN}

Este escrito midió, mediante modelos de econometría espacial con datos de corte transversal, la relación entre el Valor Agregado Municipal Per-cápita (VAMP) y El Índice de Desempeño Fiscal (IDF) en los municipios colombianos para el periodo 2011-2016. La metodología empleada inició probando la existencia de autocorrelación espacial mediante los estadísticos $I$ de Moran y $c$ de Geary; posteriormente, se estimaron modelos espaciales de relación sustantiva y residual. Los resultados obtenidos demostraron la existencia de autocorrelación espacial y la presencia de una relación positiva entre IDF y VAMP. Las principales conclusiones obtenidas indican que es necesario generar mecanismos que permitan mayor homogenización de la producción municipal mediante la liberalización del mercado y la desconcentración del poder de las entidades territoriales. También, es indispensable que los gobiernos municipales propendan por manejar niveles de gasto adecuados y austeros que no entorpezcan el crecimiento económico de su territorio. Las conclusiones mencionadas son útiles como herramienta de información en la toma de decisiones, para hacedores de política económica a cualquier nivel territorial.

\section{ABSTRACT}

This work measured the relationship between the Municipal Added Value Per-Capita (VAMP) and The Fiscal Performance Index (IDF) in Colombian municipalities in 2011-2016 using Spatial Econometrics models with cross-sectional data. The methodology employed began by testing for the existence of spatial autocorrelation using Moran's $I$ and Geary's c statistics; subsequently, spatial models of substantive and residual relationships were estimated. The results obtained demonstrated the spatial autocorrelation existence and a positive relationship between IDF and VAMP. The main conclusions obtained indicate that it is necessary to generate mechanisms that allow greater homogenization of municipal production through market liberalization and decentralization of the power of territorial entities. It is also essential that municipal governments strive to manage adequate and austere spending levels that do not hinder the economic growth of their territory. The mentioned conclusions are useful as an information tool for decision-making for economic policymakers at any territorial level. 


\section{EL DESEMPEÑO FISCAL MUNICIPAL Y SU INFLUENCIA EN EL CRECIMIENTO ECONÓMICO DE LOS MUNICIPIOS COLOMBIANOS}

\section{INTRODUCCIÓN}

La relación entre el desempeño fiscal de un gobierno a cualquier nivel territorial y el crecimiento económico de dicha región reviste una importancia notoria en cualquier estudio económico. El Estado es un actor fundamental al intervenir la economía extrayendo recursos del sector productivo (principalmente trabajadores y empresas) e inyectando recursos mediante gasto público. En general, se supone que un adecuado manejo del recaudo y el gasto público generan crecimiento para la economía. Dos trabajos destacados sobre este tema son:

- "Descentralización fiscal en América Latina. Impacto social y determinantes", de Pinilla et al. (2014): En este trabajo se pretendía verificar, para 17 países de Latinoamérica, la relación positiva entre los incrementos en la descentralización del Estado y las mejoras en el bienestar social de la sociedad. Para el logro de este objetivo se planteó un modelo en el que se define como variable endógena el resultado social de una política (RS) en función de las características estructurales de una economía $\theta$. Los autores concluyen que la descentralización siempre tiene relación positiva con los resultados sociales; generalmente, esta relación se explica en los niveles de desarrollo urbano, nivel de ingresos, nivel de democracia y grado de burocracia central, porcentaje de impuestos directos e infraestructuras sociales (Pinilla et al., 2014). Dicho trabajo relaciona el buen manejo estatal a diversas variables y el bienestar social; sin embargo, el enfoque desarrollado se fundamenta en variables sociales y desconoce el efecto del territorio en las estimaciones realizadas.

- "Crecimiento económico y política fiscal: una revisión crítica de la literatura", de Chamorro (2017): "El trabajo tiene como finalidad realizar una revisión de la literatura, teórica y empírica, sobre la relación entre la política fiscal y el crecimiento económico" (p. 79). En general, se busca contrastar los postulados de los modelos de crecimiento endógeno que explican cómo la intervención estatal puede impulsar el crecimiento económico. Los principales enfoques teóricos analizados son: 1. El modelo de Barrio, 2. El modelo de Bajo Rubio y 3. El modelo de Milbourne, Otto y Voss. Al contrastar los postulados de estos modelos con la evidencia empírica, se obtienen como principales conclusiones del trabajo que los modelos de crecimiento endógenos son certeros al afirmar que el gasto público afecta el crecimiento económico en el largo plazo, pero no todo el gasto público genera el mismo efecto; es decir, no hay efectos homogéneos en el crecimiento económico ante la realización de gasto público. La principal limitante de lo presentado por Chamorro (2017) es la no realización de estimaciones propias sobre la relación teórica establecida.

A pesar de que el tema de la relación entre el desempeño fiscal y el comportamiento de variables de crecimiento económico ha sido estudiado, a la fecha no se han realizado modelos econométricos de corte transversal que midan esta relación y que, además, incluyan el efecto del territorio en los resultados. Por tanto, el principal objetivo de este trabajo es medir, mediante modelos de econometría espacial con datos de corte transversal, la relación entre el Valor Agregado Municipal Per-cápita (VAMP) y El Índice de Desempeño Fiscal (IDF) en los municipios colombianos para el periodo 2011-2016. En el estudio se verifica la existencia de autocorrelación espacial y se construyen modelos de relación sustantiva y residual.

La hipótesis fundamental de este trabajo considera la racionalización del gasto público y del recaudo de impuestos a nivel municipal, que en conjunto suponen un mejor desempeño fiscal y permiten mejores resultados en el VAMP. Se presume que la ubicación geográfica de un municipio influye en los valores de las variables IDF y VAMP.

\section{METODOLOGÍA}

\section{Variables y datos}

El marco metodológico de este trabajo se resume en un escrito producto de una investigación aplicada de corte analítico y cuantitativo.

La base de datos utilizada fue tomada del GeoPortal del Departamento Administrativo Nacional de Estadística (DANE) (2019); los datos obtenidos fueron georreferenciados y corresponden a las variables VAMP, expresadas en pesos colombianos corrientes, e IDF. El periodo trabajado para las dos variables comprende desde el año 2011 al año 2016, con periodicidad anual.

El VAMP se obtiene al dividir el Valor Agregado Municipal (VAM) con el número de habitantes del 
municipio en cuestión (POB). Esto es:

$$
V A M P_{i}=\frac{V A M_{i}}{P O B_{i}}
$$

Donde el subíndice $i$ representa al $i$ - ésimo municipio de los 1127 municipios colombianos. La ley 1551 de 2012 responsabiliza al DANE del cálculo del Indicador de Importancia Económica Municipal (IIEM), que permite la categorización de municipal de acuerdo a las escalas establecidas en dicha ley (Ley 1551, 2012). En ese contexto, el VAM es un componente del IIEM, estimado por el DANE, que se obtiene al restar el valor agregado de la producción bruta municipal a los consumos intermedios empleados (DANE, 2016). Lo anterior implica que el $V A M P_{i}$ es un indicador económico del nivel de ingreso promedio de los habitantes de cada municipio.

El IDF surge a raíz de la ley 617 de 2000 en la cual se prioriza el logro de la viabilidad financiera de los municipios, fundamentándose en la racionalidad de los gastos administrativos municipales y en el impulso a la generación de recursos propios (Ley 617, 2000). El Departamento Nacional de Planeación - DNP (2019) estima el IDF como un índice que incluye los componentes municipales:

- Autofinanciación de los gastos de funcionamiento,

- Respaldo del servicio de la deuda,

- Dependencia de las transferencias de la nación y regalías,

- Generación de recursos propios,

- Magnitud de la inversión y

- Capacidad de ahorro. (p. 2)

La variable IDF toma valores entre cero (0) y cien (100), siendo cien el mejor resultado posible para un municipio. El dato obtenido clasifica a los municipios de acuerdo a las siguientes cinco categorías o niveles de desempeño fiscal que el DNP (2019) establece así:

1. Solvente: $I D F>80$

2. Sostenible $70<I D F \leq 80$

3. Vulnerable $60<I D F \leq 70$

4. Riesgo $40<I D F \leq 60$

5. Deterioro $I D F \leq 40$ (p. 1).

Los niveles (rangos) permiten clasificar a los gobiernos municipales como débiles o avanzados en el cumplimiento

\footnotetext{
${ }^{1}$ La palabra espacial se refiere al territorio, para el caso de estudio a los municipios.
}

del objetivo de viabilidad financiara territorial (DNP, 2019).

\section{Estadísticos I de Moran y c de Geary}

Los estadísticos globales $I$ de Moran y $c$ de Geary permiten identificar la presencia de autocorrelación en datos espaciales. Por tanto, la autocorrelación espacial responde al estudio de la dependencia espacial en datos georreferenciados, siendo este un proceso estocástico espacial ${ }^{1}$ definido como:

$$
y_{i}, i \in D \quad(2)
$$

donde $D$ representa un conjunto finito y discreto de localizaciones geográficas. Lo anterior hace evidente que cada variable aleatoria debe ser georreferenciada, por lo cual la autocorrelación espacial se expresa formalmente como:

$$
\begin{aligned}
\operatorname{COV}\left[y_{i}, y_{j}\right]= & E\left[y_{i}, y_{j}\right]-E\left[y_{i}\right] \cdot E\left[y_{j}\right] \\
& \neq 0
\end{aligned}
$$

Cuando $i \neq j$. Es decir, la autocorrelación espacial se mide como la covarianza de una variable aleatoria con observaciones individuales $y_{i}$ e $y_{j}$, siendo $i, j$ observaciones individuales (ubicaciones) de la variable $y$ (Anselin, 2001).

El estudio de la autocorrelación espacial depende de la construcción de la matriz de pesos espaciales o matriz $W$ definida así:

$$
W=\left[\begin{array}{ccc}
0 & \cdots & w_{1 N} \\
\vdots & \ddots & \vdots \\
w_{N 1} & \cdots & 0
\end{array}\right]
$$

Por tanto, $W$ es una matriz cuadrada de orden $n \times n$, con $w_{i j}$ pesos espaciales que toman el valor de uno (1) cuando las regiones 2 son consideradas vecinas, y cero (0) en otro caso. La diagonal principal de la matriz $W$ es cero, al considerarse que ninguna región es vecina de sí misma (Moreno y Vayá, 2002).

Dos de los estadísticos globales más utilizados en la medición de la autocorrelación espacial son el I de Moran y el c de Geary. Los dos se estiman con base en la matriz $W$, la cual, para el presente escrito, se estimó para cinco (5) vecinos cercanos e incluyó a los municipios "islas" o sin vecinos continuos.

\section{Estadístico I de moran}

${ }^{2}$ Región en este estudio es sinónimo de municipio. 
Este estadístico se expresa como:

$I=\frac{n}{S_{0}} \frac{\sum_{i} \sum_{j}\left(y_{i}-\bar{y}\right) w_{j i}\left(y_{j}-\bar{y}\right)}{\sum_{i=1}^{N}\left(y_{i}-\bar{y}\right)^{2}}$

donde $S_{0}=\sum_{i=1} \sum_{j=1} 1^{\prime} W 1$, con 1 como vector $(n \times 1)$ de unos, y con $\bar{y}$ representando el promedio de la muestra de datos. Valores positivos (mayores a su valor esperado) del I de Moran implican autocorrelación espacial positiva, y valores negativos (menores a su valor esperado) de este indicador muestran autocorrelación espacial negativa (Herrera, 2015).

\section{Estadístico de Geary}

Este indicador viene formulado por la expresión:

$$
=\frac{n-1}{2 S_{0}} \frac{\sum_{i=1}^{n} \sum_{j=1}^{n} w_{i j}\left(y_{i}-\bar{y}\right)^{2}}{\sum_{i=1}^{N}\left(y_{i}-\bar{y}\right)^{2}}
$$

Cuando $c$ es menor que el valor esperado, existe autocorrelación espacial positiva, y cuando $\mathrm{c}$ es mayor al valor esperado se evidencia autocorrelación espacial negativa (Herrera, 2015).

\section{Modelos de econometría espacial con datos de corte transversal}

Los modelos econométricos espaciales lineales son muy útiles para estimar relaciones de dependencia entre variables, con datos de corte transversal en unidades espaciales. En general, un modelo econométrico de este tipo clarifica el grado de dependencia espacial entre variables endógenas y exógenas, para las cuales se ha determinado estructura espacial (Xu y Lee, 2018). En este trabajo, los modelos econométricos estimados han sido la principal herramienta de medición para el logro del objetivo principal del trabajo. En todos los modelos se asume como variable dependiente el VAMP y como variable independiente el IDF; esto de acuerdo a la hipótesis que se pretendía contrastar. Los modelos estimados se han trabajado con datos de corte transversal y son:

- Modelo lineal clásico,

- Modelo de rezago espacial,

- Modelo de error espacial,

- Modelo SARAR,

- Modelo espacial de Durbin y

- Modelo de Cliff-Ord

\section{Modelo lineal clásico}

Este modelo se estima mediante Mínimos Cuadrados Ordinarios (MCO) y se basa en la estructura básica del modelo de regresión lineal. Además, asume los supuestos de normalidad, independencia, funcionalidad $y$ especificación, es decir:

con

$$
y=X \beta+\mu
$$

$$
\mu \sim\left(0, \sigma^{2} I_{n}\right)
$$

Donde $y$ y $X$ representan las variables independiente y dependientes respectivamente, $y$ ilustra un vector $(n \times 1)$ y $X$ una matriz $(n \times k), \beta$ representa un vector de parámetros desconocidos $(k \times 1)$ y $\mu$ es el término del error con dimensión $(n \times 1)$ (Anselin y Florax, 1995).

El modelo obtenido contrasta la hipótesis nula de no autocorrelación espacial, de igual forma que los contrastes globales de autocorrelación. Por lo anterior, la estructura espacial del modelo puede probarse mediante la prueba I de Moran sobre los residuales del modelo obtenido, o

\begin{tabular}{|c|c|c|c|}
\hline Prueba & Especificación & $\begin{array}{c}\text { Hipótesis Nula } \\
H_{o}\end{array}$ & Observaciones \\
\hline I de Moran & $I=\frac{n}{S_{0}} \frac{\hat{\mu}^{\prime} W \hat{\mu}}{\hat{\mu}^{\prime} \hat{\mu}}$ & $\begin{array}{l}\text { No } \\
\text { autocorrelación } \\
\text { espacial }\end{array}$ & $\begin{array}{l}\hat{\mu}: \text { Vector de } \\
\text { residuos } \\
n: \text { Número de } \\
\text { observaciones } \\
S_{0}: \text { Sumatoria de los } \\
\text { elementos de la } \\
\text { matriz } W .\end{array}$ \\
\hline $\begin{array}{lr}\text { Contraste } & \text { LM } \\
\text { para } & \text { detectar }\end{array}$ & $L M_{E R R O R}=\frac{1}{T_{1}}\left(\frac{\hat{\mu}^{\prime} W \hat{\mu}}{\sigma^{2}}\right)^{2} \underset{a s}{\sim} \chi_{(1)}^{2}$ & $\begin{array}{l}\text { Parámetro } \\
\text { espacial }\end{array}$ & $\begin{array}{l}\mu=\rho W \mu+\varepsilon \\
W: \text { Matriz de pesos }\end{array}$ \\
\hline
\end{tabular}
mediante el conjunto de pruebas del Multiplicador de Lagrange (LM). En la tabla 1 se resumen estos contrastes:

Tabla 1. Pruebas para la estructura espacial del modelo lineal clásico 
Alexander Carvajal y Oscar David Andrés Julián López-Camargo

\begin{tabular}{|c|c|c|c|}
\hline Prueba & Especificación & $\begin{array}{c}\text { Hipótesis Nula } \\
H_{o}\end{array}$ & Observaciones \\
\hline $\begin{array}{l}\text { autocorrelación } \\
\text { espacial en el error }\end{array}$ & & $\begin{array}{l}\text { autorregresivo } \\
\text { es cero } \rho=0\end{array}$ & $\begin{array}{l}\text { espaciales no } \\
\text { estocástica }(n \times n) \\
\varepsilon \text { : Vector de } \\
\text { innovaciones con } \\
\left(0, \sigma^{2} I\right) . \\
T_{1} \\
=\operatorname{tr}\left[\left(W^{\prime}+W\right) W\right] \\
\hat{\mu}: \operatorname{Residuos} M C O \\
\hat{\sigma}^{2}=\hat{\mu}^{\prime} \frac{\hat{\mu}}{n}\end{array}$ \\
\hline $\begin{array}{l}\text { Contraste LM } \\
\text { para el modelo } \\
\text { con estructura } \\
\text { espacial } \\
\text { sustantiva } \\
\text { (modelo de rezago } \\
\text { espacial) }\end{array}$ & $L M_{L A G}=\frac{1}{T_{1}} \frac{\left(\frac{\hat{\mu}^{\prime} W y}{\sigma^{2}}\right)^{2}}{n \hat{\jmath} \lambda \beta} \underset{a s}{\sim} \chi_{(1)}^{2}$ & $\begin{array}{l}\text { Parámetro } \\
\text { espacial } \\
\text { autorregresivo } \\
\text { es cero } \lambda=0\end{array}$ & $\begin{array}{l}y=\lambda W y+X \beta+\mu \\
W y: \text { Rezago espacial } \\
\text { de la variable } \\
\text { dependiente. } \\
\hat{J} \lambda \beta= \\
1 / n \hat{\sigma}^{2}[M(W X \hat{\beta})+ \\
\left.T_{1} \hat{\sigma}^{2}\right] \operatorname{con} M=I- \\
X\left(X^{\prime} X\right)^{-1} X^{\prime}\end{array}$ \\
\hline $\mathrm{LM}^{*}$ error & $\begin{array}{l}L M_{E R R O R}^{*} \\
=\frac{\left[\left(\frac{\hat{\mu}^{\prime} W \hat{\mu}}{\hat{\sigma}^{2}}\right)-T_{1}(n \hat{\jmath} \lambda \beta)^{-1}\left(\frac{\hat{\mu}^{\prime} W \hat{\mu}}{\hat{\sigma}^{2}}\right)\right]^{2}}{\left[T_{1}-T_{1}^{2}(n \hat{\jmath} \lambda \beta)^{-1}\right]} \sim{ }_{a s} \chi_{(1)}^{2}\end{array}$ & $\begin{array}{l}\text { Parámetro } \\
\text { espacial } \\
\text { autorregresivo } \\
\text { es cero } \rho=0\end{array}$ & $\begin{array}{l}\text { Soluciona } \\
\text { problema } \\
L M_{E R R O R} \text {, el cual } \\
\text { detecta } \\
\text { autocorrelación } \\
\text { espacial debido a la } \\
\text { presencia de un } \\
\text { rezago espacial de la } \\
\text { variable endógena } \\
W y\end{array}$ \\
\hline LM*lag & $L M_{L A G}^{*}=\frac{\left[\left(\frac{\hat{\mu}^{\prime} W \hat{\mu}}{\hat{\sigma}^{2}}\right)-\left(\frac{\hat{\mu}^{\prime} W \hat{\mu}}{\hat{\sigma}^{2}}\right)\right]^{2}}{n \hat{J} \lambda \beta-T_{1}} \sim_{a s} \chi_{(1)}^{2}$ & $\begin{array}{l}\text { Parámetro } \\
\text { espacial } \\
\text { autorregresivo } \\
\text { es cero } \lambda=0\end{array}$ & $\begin{array}{l}\text { Soluciona } \\
\text { problema } \\
L M_{L A G} \text { el el cual } \\
\text { detecta } \\
\text { autocorrelación } \\
\text { espacial debido a } \\
\text { que el termino de } \\
\text { error contiene un } \\
\text { rezago espacial } W \mu\end{array}$ \\
\hline
\end{tabular}

Fuente: elaboración propia basada en Anselin y Florax (1995) y Herrera (2015).

Herrera (2015) resume la interpretación de los resultados de los contrastes mostrados en la tabla $1 \mathrm{y}$, de acuerdo con esto, la selección del mejor modelo espacial, a saber:

- $\quad \mathrm{Si} I$ de Moran rechaza $\mathrm{H}_{0}$ Evidencia a favor de inclusión de elementos espaciales

- $\quad$ Si LM ERROR y LMERROR rechazan $\mathrm{H}_{0}$ Evidencia a favor de un modelo de error espacial (SEM)

- $\quad \mathrm{Si} \mathrm{LM}_{\mathrm{LAG}}$ y $\mathrm{LM}_{\mathrm{LAG}}^{*}$ rechazan $\mathrm{H}_{0}$ Evidencia a favor de un modelo de rezago espacial (SLM)
- $\quad$ Si no se rechaza $\mathrm{H}_{0}$ bajo ninguno de los contrastes Evidencia a favor del modelo lineal general no espacial.

- $\quad \mathrm{Si}$ ambos contrastes robustos $\mathrm{LM}_{\mathrm{ERROR}}^{*}$ y $\mathrm{LM}_{\mathrm{LAG}}^{*}$ rechazan $\mathrm{H}_{0}$ Entonces se deberán incorporar elementos espaciales en la parte sistémica (Wy) y aleatoria $(W \mu)$ (p. 26).

Modelo de rezago espacial (SLM), Modelo de error espacial (SEM), Modelo SARAR, Modelo espacial de Durbin y Modelo de Cliff-Ord. Para este trabajo, estos 
modelos se estiman mediante el algoritmo de máxima verosimilitud y se resumen así:

\section{SLM}

El modelo viene dado por:

$$
y_{i t}=\lambda W y_{i t}+X_{i t} \beta+\mu
$$

La relación espacial viene dada por la variable endógena $y$ rezagada y, por tanto, $\rho=0$ (Urrego et al., 2016).

\section{SEM}

La especificación del modelo es:

donde

$$
y_{i t}=X_{i t} \beta+\mu_{i t}
$$

$$
\mu_{i t}=\rho W \mu_{i t}+\varepsilon_{i t}
$$

En este modelo el rezago espacial es el término de error, por lo cual la relación espacial depende del "contagio" generado por los choques exógenos propagados entre las unidades vecinas. El modelo parte del supuesto $\lambda=0$ (Urrego et al., 2016).

\section{SARAR}

Este modelo supone rezagos espaciales en la variable dependiente y en el término del error; esto es, los choques aleatorios en las unidades vecinas y la relación directa entre la variable endógena y su rezago espacial generan, conjuntamente, la relación espacial. El modelo parte del supuesto $\gamma=0$ (Urrego et al., 2016). La especificación del modelo es:

$$
\begin{gathered}
y_{i t}=\lambda W y_{i t}+X_{i t} \beta+\mu_{i t} \\
\mu_{i t}=\rho W \mu_{i t}+\varepsilon_{i t}
\end{gathered}
$$

\section{Durbin}

Este modelo supone rezagos espaciales tanto en la variable endógena como en las exógenas, y que $\gamma=0$. Se plantea así la existencia de una relación espacial explicada por las características individuales de las unidades vecinas, es decir, las intervenciones sobre las variables explicativas generan y propagan los choques espaciales (Urrego et al., 2016). Su formulación se estructura así:

$$
\begin{gathered}
y_{i t}=\lambda W y_{i t}+X_{i t} \beta+W X_{i t} \gamma \\
+\mu_{i t}
\end{gathered}
$$

\section{Cliff-Ord}

En este modelo se incluye dependencia sustantiva, residual y local, explicada por las variables independientes del modelo (Herrera, 2015). Su representación analítica es:

$$
\begin{gathered}
y_{i t}=\lambda W y_{i t}+X_{i t} \beta+W X_{i t} \gamma+\mu_{i t} \\
\mu_{i t}=\rho W \mu_{i t}+\varepsilon_{i t}
\end{gathered}
$$

Los modelos presentados se estimaron para cada uno de los años del periodo en estudio (2011-2016). En las estimaciones se consideró como variable dependiente el VAMP y como variable independiente el IDF. La utilización de estos modelos permite establecer la existencia de relación residual y/o sustantiva. La relación sustantiva se refiere a variables correlacionadas espacialmente, es decir, el rezago de una variable (generalmente la endógena) explica el comportamiento de dicha variable, y la relación residual indica que la correlación espacial debe incluir el término del error (residuo) (Alonso, 2015).

\section{RESULTADOS}

Para la correcta estimación de los estadísticos I de Moran, $c$ de Geary y de los modelos de regresión explicados en la sección previa se construyó la matriz de contigüidad para cinco vecinos cercanos, de dimensiones $1127 \times 1127$, obteniéndose 5635 links de un total de 1270129 links posibles. En la figura 1 se puede observar la representación gráfica de la matriz de contigüidad. 


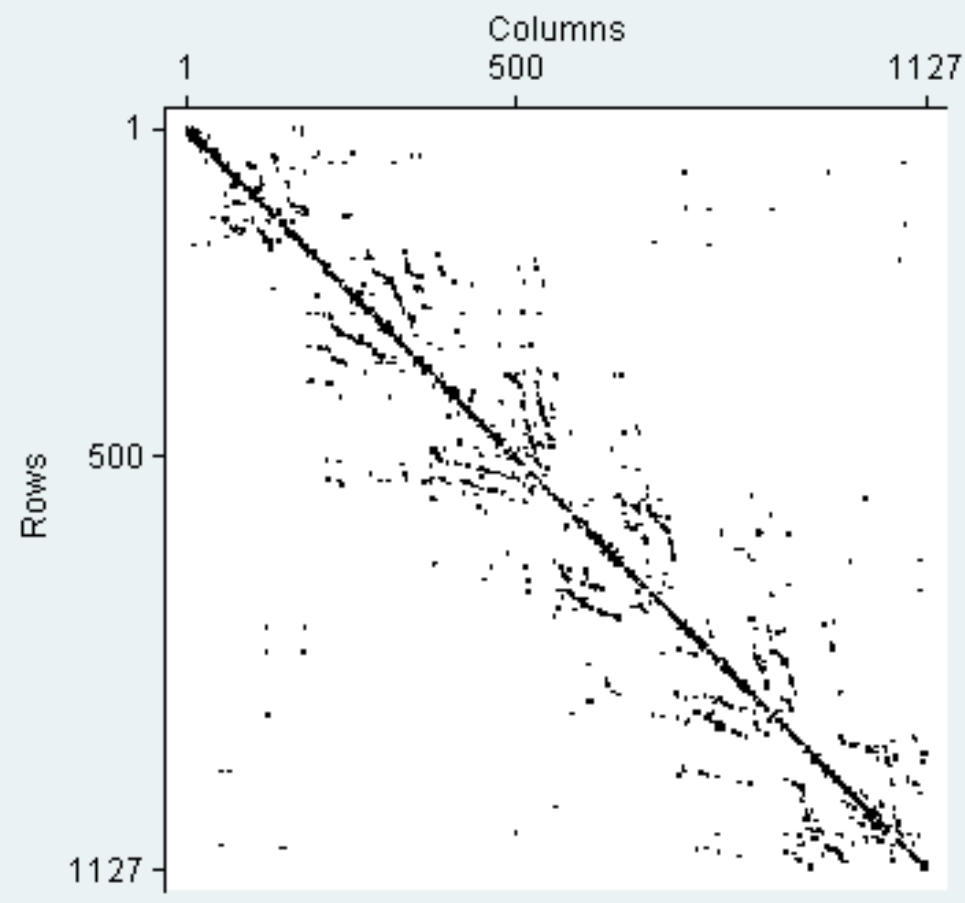

Figura 1. Matriz de contigüidad espacial municipios de Colombia

Fuente: elaboración propia basada en datos del DANE

La tabla 2 muestra los resultados de la estimación de los

VAMP e IDF para cada año del periodo estudiado.

estadísticos $I$ de Moran y $c$ de Geary de las variables

Tabla 2. I de Moran y $c$ de Geary

\begin{tabular}{|l|l|l|l|l|l|l|l|l|}
\hline \multirow{2}{*}{ Año/Estimador } & \multicolumn{4}{|c|}{ VAMP } & \multicolumn{4}{c|}{ IDF } \\
\cline { 2 - 9 } & $\begin{array}{c}\text { I de } \\
\text { moran }\end{array}$ & $\boldsymbol{P}$-valor & $\boldsymbol{C}$ de Geary & $\boldsymbol{p}$-valor & $\begin{array}{c}\text { I de } \\
\text { moran }\end{array}$ & P-valor & $\begin{array}{c}\text { Cde } \\
\text { Geary }\end{array}$ & $\boldsymbol{p}$-valor \\
\hline 2011 & 0,209 & 0,000 & 0,670 & 0,000 & 0,378 & 0,000 & 0,618 & 0,000 \\
\hline 2012 & 0,125 & 0,000 & 0,609 & 0,000 & 0,405 & 0,000 & 0,598 & 0,000 \\
\hline 2013 & 0,105 & 0,000 & 0,618 & 0,000 & 0,400 & 0,000 & 0,595 & 0,000 \\
\hline 2014 & 0,117 & 0,000 & 0,637 & 0,000 & 0,371 & 0,000 & 0,633 & 0,000 \\
\hline 2015 & 0,141 & 0,000 & 0,651 & 0,000 & 0,344 & 0,000 & 0,661 & 0,000 \\
\hline 2016 & 0,174 & 0,000 & 0,687 & 0,000 & 0,325 & 0,000 & 0,673 & 0,000 \\
\hline
\end{tabular}

Fuente: elaboración propia

De acuerdo con la tabla 2 sobre los estadísticos $I$ de Moran y $c$ de Geary, se puede afirmar que con un 0,95 (95\%) de confianza se rechaza la hipótesis nula de ausencia de autocorrelación espacial. Esto se puede verificar al observar que todos los $p$ - valor son menores al 0,05 (5 $\%)$. Además, cada valor del $I$ de Moran indica el grado de asociación de la variable en forma porcentual; por ejemplo, para 2011 la variable VAMP presenta un valor de autocorrelación positiva cercano al 0,21 (21\%). El $c$ de Geary se puede leer análogamente y se debe recordar que los valores altos implican lecturas similares a valores bajos del $I$ de Moran.

Además, con los resultados mostrados en la tabla 2 se puede identificar la relación de la autocorrelación de acuerdo a los valores del I de Moran para las dos variables en estudio. Esto es, para la variable VAMP existe autocorrelación global positiva en todos los años, con valores entre el 0,1 y el 0,2 (10\% y $20 \%$, respectivamente). La variable IDF presenta autocorrelación global positiva, pero con valores entre 3 y 0,4 (30 \% y $40 \%)$ para todos los años. La autocorrelación positiva implica comportamientos 
EL DESEMPEÑO FISCAL MUNICIPAL Y SU INFLUENCIA EN EL CRECIMIENTO ECONÓMICO DE LOS MUNICIPIOS

COLOMBIANOS

similares entre una unidad espacial y su vecino más cercano. Para este estudio, la unidad espacial es cada municipio.

Los resultados de la estimación de los modelos de regresión espacial propuestos se detallan a continuación.

\section{Modelo lineal clásico}

En la tabla 3 se muestran los resultados de la estimación de los modelos lineales clásicos y los diagnósticos de los residuos para cada modelo.

Tabla 3. Resultados modelo lineal clásico

\begin{tabular}{|c|c|c|c|c|c|c|c|c|}
\hline \multirow[b]{2}{*}{ Año } & \multicolumn{3}{|c|}{ Modelo lineal clásico } & \multicolumn{5}{|c|}{ Diagnóstico de residuos } \\
\hline & Constante & $\beta_{1}$ & valor $F$ & I Moran & $L M_{E R R O R}$ & $L M_{E R R O R}^{*}$ & $L M_{L A G}$ & $L M_{L A G}^{*}$ \\
\hline 2011 & $2,85 e+06$ & $1750,739 * * *$ & $8,78^{* * *}$ & $11,2 * * *$ & $124,8 * * *$ & $2,0 \mathrm{e}+12 * * *$ & $137,3 * * *$ & $2,0 \mathrm{e}+12 * * *$ \\
\hline 2012 & $1,05 \mathrm{e}+07 * * *$ & 33,6 & 0,05 & $4,86 * * *$ & $22,46 * * *$ & 0,034 & $22,6 * * *$ & 0,024 \\
\hline 2013 & $1,11 \mathrm{e}+07 * * *$ & 38,4 & 0,05 & $5,55 * * *$ & $29,793 * * *$ & 0,107 & $29,7 * * *$ & 0,085 \\
\hline 2014 & $4,77 \mathrm{e}+06^{* *}$ & $996,9 * * *$ & $7,88 * * *$ & $7,24 * * *$ & $51,47 * * *$ & $4,7 \mathrm{e}+12 * * *$ & $64,2 * * *$ & $4,7 \mathrm{e}+12 * * *$ \\
\hline 2015 & $5,20 \mathrm{e}+06^{* *}$ & $975,0 * * *$ & $9,06 * * *$ & $6,36 * * *$ & $39,59 * * *$ & $16,9 * * *$ & $43,6 * * *$ & $20,9 * * *$ \\
\hline 2016 & $1,23 \mathrm{e}+07 * * *$ & 5,02 & 0,00 & $6,51 * * *$ & $41,08 * * *$ & 0,036 & $41,0 * * *$ & 0,032 \\
\hline
\end{tabular}

Fuente: elaboración propia basada en datos del DANE.

Nota: $* * *$ denotan significancia (rechazo de la hipótesis nula) al 0,01 (1\%); ** denotan significancia al 0,95 (95\%) y * denota significancia al 0,9 (90\%). La ausencia de asteriscos denota no significancia. Cuando la constante no es significativa, los valores del modelo corresponden a la estimación sin constante.

De acuerdo a los valores entregados en la tabla tres se puede concluir que:

- En el año 2011 debe estimarse un modelo SARAR.

- En el año 2012 es evidente que se deben incluir elementos espaciales, pero los contrastes robustos no permiten tener claridad sobre el mejor modelo a estimarse. Por ello, se debe estimar el modelo SLM y el modelo SEM.

- En el año 2013 es evidente que se deben incluir elementos espaciales, pero los contrastes robustos no permiten tener claridad sobre el mejor modelo a estimarse. Por ello, se debe estimar el modelo SLM y el modelo SEM.

- En el año 2014 debe estimarse un modelo SARAR.

- En el año 2015 debe estimarse un modelo SARAR.

- En el año 2016 es evidente que se deben incluir elementos espaciales, pero los contrastes robustos no permiten tener claridad sobre el mejor modelo a estimarse. Por ello, se debe estimar el modelo SLM y el modelo SEM.
Los resultados de los años 2012, 2013 y 2016 se explican por la no significancia de los valores de la pendiente y de la prueba $F$.

\section{SLM}

Los modelos SLM sugeridos por el diagnostico de residuos delo modelo lineal clásico entregaron las siguientes estimaciones:

Año 2012

$$
\begin{gathered}
V A M P_{i}=7.660 .000+0.28 \mathrm{WVAM} P_{i} \\
+40.8 I D F_{i}+\mu_{i}
\end{gathered}
$$

Año 2013

$$
\begin{gathered}
V A M P_{i}=7.610 .000+0.33 \mathrm{WVAM} P_{i} \\
+46.6 I D F_{i}+\mu_{i}
\end{gathered}
$$

Año 2016

$$
\begin{gathered}
V A M P_{i}=8.610 .000+0.3 W V A M P_{i} \\
+11.26 I D F_{i}+\mu_{i}
\end{gathered}
$$

\section{SEM}

Los modelos SEM sugeridos por el diagnostico de residuos delo modelo lineal clásico entregaron las siguientes estimaciones:

Año 2012

$$
\begin{gathered}
V A M P_{i}=10.700 .000+53.146 I D F_{i} \\
+\mu_{i}
\end{gathered}
$$

con

$$
\mu_{i}=0.28 W \mu_{i}+\varepsilon_{i}
$$


Año 2013

$$
\begin{gathered}
V A M P_{i}=11.300 .000+66.65 I D F_{i} \\
+\mu_{i}
\end{gathered}
$$

con

$$
\mu_{i}=0.32 W \mu_{i}+\varepsilon_{i}
$$

Año 2016

$$
\begin{gathered}
V A M P_{i}=12.300 .000+14.72 I D F_{i} \\
+\mu_{i}
\end{gathered}
$$

con

$$
\mu_{i}=0.3 W \mu_{i}+\varepsilon_{i}
$$

\section{SARAR}

Los modelos SARAR sugeridos por el diagnostico de residuos delo modelo lineal clásico entregaron las siguientes estimaciones:

Año 2011

$$
\begin{gathered}
\operatorname{VAMP}_{i}=-2.690 .000+0.70 \mathrm{WVAMP} \\
\text { con } \quad+1010.325 I D F_{i}+\mu_{i} \\
\mu_{i}=-0.43 W \mu_{i}+\varepsilon_{i}
\end{gathered}
$$

Año 2014

$$
\begin{aligned}
& V A M P_{i}=4.120 .000+0.22 \mathrm{WVAMP} P_{i} \\
& +722.31 I D F_{i}+\mu_{i}
\end{aligned}
$$

con

$$
\mu_{i}=0.14 W \mu_{i}+\varepsilon_{i}
$$

Año 2015

$$
\begin{aligned}
& V A M P_{i}=4.640 .000+0.23 \mathrm{WVAM} P_{i} \\
& +676.61 I D F_{i}+\mu_{i}
\end{aligned}
$$

con

$$
\mu_{i}=0.15 W \mu_{i}+\varepsilon_{i}
$$

Adicionalmente a los modelos sugeridos por el diagnóstico de residuos del modelo lineal clásico, se estimaron los modelos de Durbin y de Cliff-Ord para todos los años del periodo en estudio. Esto con el objetivo de mejorar la robustez de las estimaciones realizadas al usar modelos más complejos.

\section{Modelo de Durbin}

Año 2011

$$
\begin{aligned}
V A M P_{i}=- & 11.900 .000+0.44 W V A M P_{i} \\
& +870.86 I D F_{i} \\
& +2078.38 W I D F_{i}+\mu_{i}
\end{aligned}
$$

Año 2012

$$
\begin{aligned}
V A M P_{i}=7.750 .000 & +0.28 W V A M P_{i} \\
& +67.41 I D F_{i}-41.51 W I D F_{i} \\
& +\mu_{i} \quad(25)
\end{aligned}
$$

Año 2013

$$
\begin{aligned}
V A M P_{i}=7.790 .000 & +0.32 W V A M P_{i} \\
& +92.38 I D F_{i}-71.69 W I D F_{i} \\
& +\mu_{i} \quad(26)
\end{aligned}
$$

Año 2014

$$
\begin{aligned}
V A M P_{i}=- & 9.100 .000+0.32 \mathrm{WVAMP} \\
& +696.02 I D F_{i} \\
& +1929.13 W I D F_{i}+\mu_{i}
\end{aligned}
$$

Año 2015

$$
\begin{aligned}
V A M P_{i}=- & 11.600 .000+0.32 \mathrm{WVAMP} \\
& +666.94 I D F_{i} \\
& +2355.89 W I D F_{i}+\mu_{i}
\end{aligned}
$$

Año 2016

$$
\begin{aligned}
V A M P_{i}=8.640 .000 & +0.30 \mathrm{WVAMP} P_{i} \\
& +17.74 I D F_{i}-10.13 W I D F_{i} \\
& +\mu_{i} \quad(29)
\end{aligned}
$$

\section{Modelo Cliff-Ord}

\section{Año 2011}

$$
\begin{array}{rl}
V A M P_{i}=-1 & 0.400 .000+0.68 \mathrm{WVAMP} \\
& +623.62 I D F_{i} \\
& +1665.89 W I D F_{i}+\mu_{i}
\end{array}
$$

con

Año 2012

$$
\mu_{i}=-0.42 W \mu_{i}+\varepsilon_{i}
$$

$$
\begin{aligned}
V A M P_{i}=9.040 .000 & +0.16 \mathrm{WVAMP} P_{i} \\
& +62.66 I D F_{i}-26.64 W I D F_{i} \\
& +\mu_{i} \quad(31)
\end{aligned}
$$

con

$$
\mu_{i}=0.16 W \mu_{i}+\varepsilon_{i}
$$

Año 2013

$$
\begin{aligned}
V A M P_{i}=9.270 .000 & +0.19 W V A M P_{i} \\
& +82.29 I D F_{i}-46.07 W I D F_{i} \\
& +\mu_{i} \quad(32)
\end{aligned}
$$

con

$$
\mu_{i}=0.19 W \mu_{i}+\varepsilon_{i}
$$

Año 2014

$$
\begin{aligned}
& V A M P_{i}=-8.980 .000+0.21 \mathrm{WVAMP} P_{i} \\
& +740.73 I D F_{i} \\
& +2056.71 W I D F_{i}+\mu_{i} \\
& \mu_{i}=0.13 W \mu_{i}+\varepsilon_{i}
\end{aligned}
$$

Año 2015 


$$
\begin{array}{rl}
V A M P_{i}=-1 & 2.000 .000+0.19 W V A M P_{i} \\
& +730.31 I D F_{i} \\
& +2572.49 W I D F_{i}+\mu_{i}
\end{array}
$$

con

$$
\mu_{i}=0.16 W \mu_{i}+\varepsilon_{i}
$$

Año 2016

$$
\begin{array}{rl}
V A M P_{i}=10 & 400.000+0.15 W V A M P_{i} \\
& +17.45 I D F_{i}-8.74 W I D F_{i} \\
& +\mu_{i} \quad \text { (35) }
\end{array}
$$

con

$$
\mu_{i}=0.16 W \mu_{i}+\varepsilon_{i}
$$

\section{DISCUSIÓN}

En este trabajo se demuestra que existen regiones que concentran los mejores valores de VAMP e IDF. Al ser el VAMP una variable que permite medir el valor monetario de la producción, es una variable de riqueza, por lo cual, es válido asumir que la riqueza se concentra en algunas regiones del país. Este planteamiento reafirma lo indicado por Ramírez et al. (2007) quienes encontraron que en Colombia existe una relación espacial con estructura centro-periferia, donde en la región noroccidental del país se concentran los departamentos con mejores desempeños económicos en términos de competitividad. Además, identificaron la región Bogotá- Cundinamarca como la de mayor concentración poblacional y productiva del país (Ramírez et al., 2007). Por otra parte, Ávila y Solano (2018) muestran que los departamentos categorizados en el nivel 4 de desempeño fiscal (peor desempeño fiscal comparativo) son los departamentos de Caquetá, Casanare, Guajira, Vichada, Guainía, Arauca, Guaviare, Putumayo, Vaupés, Amazonas y Chocó. Estos departamentos tradicionalmente se consideran periféricos en temas económicos.

Por otra parte, es claro el elevado nivel de importancia para los municipios, de implementar políticas económicas que permitan sanear sus finanzas y generar mecanismos de autofinanciación (mejorar su IDF) para impactar positivamente los niveles de producción e ingreso municipal, medidos por medio de la variable VAMP. Este postulado tiene concordancia con lo concluido por Mendoza y Campos (2015), quienes identificaron que las regiones más "prosperas" del país son aquellas que gestionan más eficientemente los recursos públicos, en especial el gasto público en infraestructura asociada al incremento de ventajas competitivas.

Finalmente, la importancia del territorio como actor de influencia sobre las relaciones de las variables es un tema que no debe descartarse ante modelos lineales no significativos, sino establecer formas funcionales que le den significancia estadística a los modelos planteados. Es así como en el trabajo "Convergencia interregional en Colombia 1990-2013. Un enfoque sobre la dinámica espacial", de Gómez y Santana (2016), la variable endógena es estimada como el logaritmo de la tasa de crecimiento del PIB y con esta variable se establecen medidas de convergencia de la producción en algunas regiones del país.

De acuerdo a los hallazgos encontrados, se plantea como discusión que:

1. Es necesario generar mecanismos que permitan mayor homogenización de la producción municipal. Si bien es cierto que es inevitable la concentración económica, se hace necesario el fomento de políticas económicas que permitan el crecimiento económico de los municipios rezagados. El postulado de los autores de este escrito es considerar que la liberalización del mercado y la desconcentración del poder de las entidades territoriales son el derrotero a seguir para esto.

2. La relación establecida entre IDF y VAMP permite a los gobiernos municipales pensar en tener niveles de gasto adecuados y austeros que no entorpezcan el crecimiento económico de su territorio.

3. Finalmente, ante el problema de la no significancia de los modelos planteados para los años 2012, 2013 y 2016 se postula, en futuras investigaciones, la necesidad de establecer modelos econométricos espaciales de corte transversal que incorporen formas funcionales en las variables endógenas y/o exógenas, esto supeditado a la coherencia con la teoría económica establecida.

\section{CONCLUSIÓN}

De acuerdo con los resultados de los estadísticos $I$ de Moran y $c$ de Geary se puede afirmar que existe autocorrelación espacial positiva para cada periodo analizado y para las dos variables en estudio VAMP e IDF. Esto es, los valores que toman el Valor Agregado percápita y el Índice de Desempeño fiscal del municipio $i-$ ésimo se asocian de forma positiva con los valores de estas variables en municipios vecinos. Por tanto, la ubicación geográfica juega un papel decisivo en la definición de los valores de las variables en estudio. 
Los modelos de regresión espaciales estimados evidencian la existencia de relación entre IDF (variable exógena) y VAMP (variable endógena). Sin embargo, la relación de dependencia no es significativa en todos los años del periodo en estudio. Además, para algunos años, la relación de dependencia es netamente sustantiva o residual, pero para otros años es sustantiva y residual. A continuación, se detalla lo evidenciado en los modelos espaciales estimados:

- En los años 2012, 2013 y 2016 existe relación positiva entre IDF y VAMP. Es decir, variaciones positivas en la variable IDF conllevan mejoras en la variable VAMP. Además, esta relación se puede explicar de forma sustantiva o incluyendo un modelo para los residuos.

- En los años 2011, 2014 y 2015 existe relación positiva entre las variables IDF y VAMP, y esta relación se explica mediante modelos que incluyen correlación sustantiva y residual.

- Los modelos de Durbin y de Cliff-Ord reafirman la relación positiva explicada en los dos puntos previos e incluyen una medida sustantiva de efecto espacial, que para los años 2012, 2013 y 2016 es negativa. En concreto, en estos años, variaciones positivas de IDF generan disminuciones de VAMP si existe vecindad del municipio í-ésimo. Esto es, el valor de la variable VAMP para el municipio $i$ - ésimo también se explica por el valor del IDF del municipio $i-1$.

- Las pruebas de significancia para los modelos aplicados a los años 2012, 2013 y 2016 no resultaron significativas conjunta ni individualmente.

\section{Declaración sobre conflictos de interés}

Los autores manifestamos que las ideas, opiniones, comentarios y conclusiones presentadas en este trabajo son independientes con respecto a la institución financiadora y de apoyo (Universidad Santo Tomás), y que durante la ejecución del trabajo o la redacción del manuscrito no han incidido intereses o valores distintos a los que usualmente tiene la investigación. Los autores no hemos incurrido en ningún conflicto de interés.

\section{REFERENCIAS BIBLIOGRÁFICAS}

Alonso, Ó. L. (2015). La econometría espacial. Un enfoque para el estudio a nivel de datos microterritoriales de la eficacia de las políticas de desarrollo rural cofinanciadas por la Unión Europea. El

caso de Asturias [tesis doctoral, Universidad de Málaga, España]. Repositorio Institucional Riuma. https://cutt.ly/zhe4FpE

Anselin, L. (2001). Spatial Econometrics. En B.

H. Baltagi. (Ed.), A Companion to Theoretical Econometrics (pp. 310-330). Blackwell Publishing. http://web.pdx.edu/ crkl/WISE/SEAUG /papers/anselin01_CTE14.pdf

Anselin, L. y Florax, R. J. (1995). New directions in spatial econometrics. Springer.

https://link.springer.com/chapter/10.100 7/978-3-642-79877-1_1

Ávila, J. A. y Solano, H. (2018). Análisis comparativo del indicador del desempeño fiscal de los departamentos categoría 4 y 1 de Colombia vigencia 2016 [trabajo de investigación, Universidad Católica de Colombia]. Repositorio Institucional RIUCaC. https://repository.ucatolica.edu.co/handl e/10983/15986

Congreso de la República de Colombia. (6 de julio de 2012). Ley 1551. Normas para modernizar la organización y el funcionamiento de los municipios. Diario $\begin{array}{lll}\text { Oficial } & \text { no. }\end{array}$ http://www.secretariasenado.gov.co/sen ado/basedoc/ley_1551_2012.html

Congreso de la República de Colombia. (6 de 
EL DESEMPEÑO FISCAL MUNICIPAL Y SU INFLUENCIA EN EL CRECIMIENTO ECONÓMICO DE LOS MUNICIPIOS COLOMBIANOS

octubre de 2000). Ley 617. Por la cual se reforma parcialmente la Ley 136 de 1994, el Decreto Extraordinario 1222 de 1986, se adiciona la Ley Orgánica de Presupuesto, el Decreto 1421 de 1993, se dictan otras normas tendientes a fortalecer la descentralización, y se dictan normas para la racionalización del gasto público nacional. Diario $\begin{array}{lll}\text { Oficial no. } & 44.188 .\end{array}$ http://www.secretariasenado.gov.co/sen ado/basedoc/ley_0617_2000.html

Chamorro, R. (2017). Crecimiento económico y política fiscal: una revisión crítica de la literatura. Ensayos de Economía, 27(51), 79-107.

http://dx.doi.org/10.15446/ede.v27n51.6 $\underline{9104}$

Departamento Administrativo Nacional de Estadística - DANE. (2016). Metodología para calcular el indicador de importancia económica municipal cuentas departamentales - CD. DANE. https://www.dane.gov.co/files/investiga ciones/fichas/Met_indicador_import_ec onomica_mpal.pdf

Departamento Administrativo Nacional de Estadística - DANE. (2019). GeoPortal. DANE.

https://geoportal.dane.gov.co/v2/

Departamento Nacional de Planeación - DNP. (2 de abril de 2019). Índice de desempeño fiscal.

http://www.anticorrupcion.gov.co/Pagin as/indice-desempeno-fiscal.aspx

Gómez, F. C. y Santana, L. (2016). Convergencia interregional en Colombia 1990-2013: un enfoque sobre la dinámica espacial. Ensayos sobre Política Económica, 34(80), 159-174. http://dx.doi.org/10.1016/j.espe.2016.03 .004

Herrera, M. H. (2015). Econometría especial usando Stata. Breve guía aplicada para datos de corte transversal. Documentos de Trabajo del Instituto de Estudios Laborales y del Desarrollo Económico, 13.

https://ri.conicet.gov.ar/handle/11336/71 $\underline{16}$

Mendoza, H. A. y Campo, J. (2015). Postura fiscal y crecimiento económico regional en Colombia. Revista Facultad de Ciencias Económicas: Investigación y Reflexión, 24(2), 29-45. http://www.scielo.org.co/scielo.php?scri $\mathrm{pt}=\mathrm{sci} \_$arttext\&pid=S0121$\underline{68052016000200003}$

Moreno, R. y Vayá, E. V. (2002). Econometría espacial: nuevas técnicas para el análisis regional. Una aplicación a las regiones europeas. Investigaciones Regionales, 1, 83-106.

http://www.redalyc.org/articulo.oa?id=2 $\underline{8900104}$

Pinilla, D. E., Jiménez, J. D. y Montero, R. 
(2014). Descentralización fiscal en América Latina. Impacto social y determinantes. Investigación Económica, 73(289), 79-110. https://doi.org/10.1016/S01851667(15)30004-7

Ramírez, J. C., Osorio, H. y Parra, R. I. (2007). Escalafón de la competitividad de los departamentos en Colombia. Naciones Unidas.

https://repositorio.cepal.org/bitstream/ha ndle/11362/4815/1/S0700110_es.pdf

Urrego, J. A., Gómez, C., Velásquez C., H. y
Valderrama, J. D. (2016). Efecto de los ingresos permanentes sobre el delito: un enfoque espacial y un caso de aplicación. Investigación Económica, 75(298), 115153.

https://doi.org/10.1016/j.inveco.2016.11 .004

Xu, X. y Lee, L. (2018). Theoretical foundations for spatial econometric research. Regional Science and Urban Economics, 76 , $1-11$. https://doi.org/10.1016/j.regsciurbeco.20 18.04.002 